\title{
SOME REMARKS ON THE PAPER BY B. G. MARSDEN "AN ATTEMPT TO RECONCILE THE DYNAMICAL AND RADAR DETERMINATIONS OF THE ASTRONOMICAL UNIT"
}

By Eugene RABE,

University of Cincinnati Observatory.

While Marsden's solution C leaves residuals with the relatively small $[v v]$ of 13.73 , it should be realized that this representation of the observations of Eros does not satisfy the fundamental principle of the least squares method, in so far as the associated value of $[v v]$ is not a minimum with respect to small arbitrary deviations from solution C. As a matter of fact, there is an infinite number of " solutions " with [vv] between the 13.73 of Marsden's solution $C$ and the 8.66 of his solution A, each of these being associated with a certain arbitrarily prescribed value of the mass of Mars and with a related mass of Earth + Moon. Of this infinite series of solutions, only solution $\mathrm{A}$ is a least squares solution in the true sense, with a minimum value of $[v v]$. This can be seen and verified as follows.

Let $\mathrm{X}_{\mathrm{A}}^{i}$ (for $\left.i=\mathrm{1}, 2, \ldots, \mathrm{1} 6\right)$ represent the individual results from solution $\mathrm{A}$ for the 16 unknowns which affect the observations equations of Eros, and $\mathrm{X}_{i}^{i}$ the corresponding results of solution C. Further, let $v_{.}^{i}$ (for $j=\mathrm{I}, 2, \ldots, 74$ ) denote the various residuals of solution $\mathrm{A}$, and $v_{\mathrm{i}}^{i}$ those of solution $\mathrm{C}$. Since the 74 differences $v_{\mathrm{C}}^{j}-v_{\mathrm{a}}^{i}$ are linear functions of the 16 differences $\mathrm{X}_{\mathrm{i}}^{i}-\mathrm{X}_{\mathrm{A}}^{i}$ between the two solutions $\mathrm{A}$ and $\mathrm{C}$, the infinite series of solutions $\mathrm{N}$, with

$$
\mathrm{X}_{\mathrm{X}}^{i}=\mathrm{X}_{\mathrm{A}}^{i}+\mathrm{N}\left(\mathrm{X}_{\mathrm{C}}^{i}-\mathrm{X}_{\mathrm{A}}^{i}\right) \quad(0<\mathrm{N}<\mathrm{I}),
$$

will leave residuals obtainable by linear interpolation according to

$$
v_{\mathrm{X}}^{j}=v_{\mathrm{A}}^{j}+\mathrm{N}\left(\boldsymbol{v}_{\mathrm{C}}^{j}-v_{\mathrm{A}}^{j}\right) \text {. }
$$

For $\mathrm{N}=\frac{\mathrm{r}}{2}$, for example, the elements of Eros and of the Earth and all the other masses and corrections represented by $\mathrm{X}_{\mathrm{x}}^{i}$ will have values 
exactly halfway between those of Marsden's solutions A and C, and the residuals will also be equal to the arithmetical means of those of $\mathrm{A}$ and $\mathrm{C}$.

Of principal interest are the $[v v]$ values associated with different values of N. Equation (2) has been used to compute the residuals associated with $\mathrm{N}=\frac{1}{4}, \frac{\mathrm{I}}{2}, \frac{3}{4}$, and also those for $\mathrm{N}=-\frac{1}{4},-\frac{\mathrm{I}}{2}$. The related square sums $[v v]$ are listed in table I, together with the associated mass values for Mars and Earth + Moon. In figure $\mathrm{I}$ the $[v v]$ have been plotted

TABLE I.

$\lceil v v\rceil$ of Eros for interpolated solutions.

\begin{tabular}{|c|c|c|c|c|}
\hline $\mathbf{N}$. & {$[v v]$} & $\frac{1}{m^{x}}$. & $\frac{1}{m_{\oplus+\mathbb{C}}}$ & Remarks. \\
\hline$-0.50 \ldots \ldots \ldots$ & 9.60 & 3123600 & 328418 & \\
\hline$-0.25 \ldots \ldots \ldots$ & 8.82 & 3106200 & 328499 & \\
\hline $0.00 \ldots \ldots \ldots$ & 8.66 & 3 o88 9ou & 328579 & Solution A \\
\hline$+0.25 \ldots \ldots \ldots$ & $9 \cdot 10$ & 307 I 800 & 328659 & \\
\hline$+0.50 \ldots \ldots \ldots$ & ro.o3 & 3 o5/1900 & 328740 & \\
\hline$+0.75 \ldots \ldots \ldots$ & 11.58 & 3038200 & 328820 & \\
\hline$+1.00 \ldots \ldots \ldots$ & 13.73 & 3 0? I 700 & 328900.5 & Solution C \\
\hline
\end{tabular}

against $\mathrm{N}$, and the curve connecting the computed points clearly exhibits the significance of solution $\mathrm{A}$ as the only true least squares solution, as far as the representation of the Eros motion is concerned. Marsden's

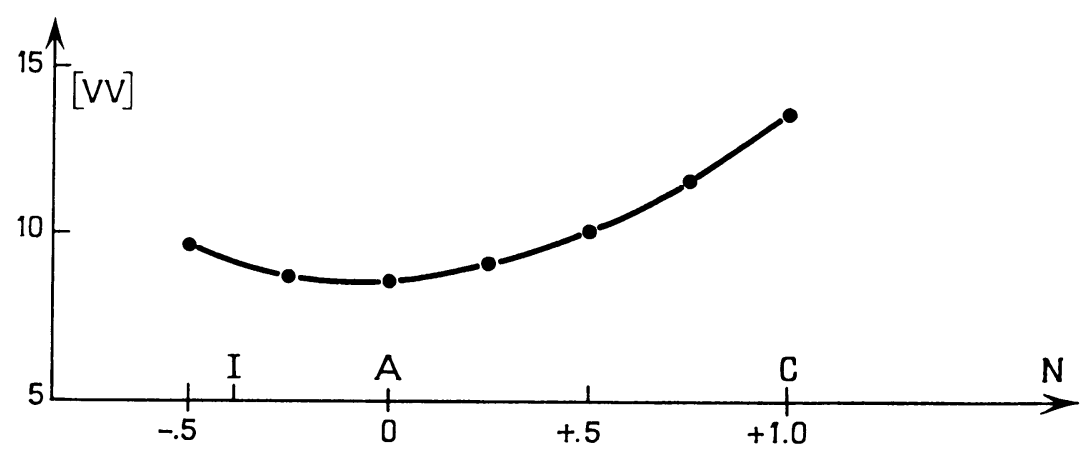

Fig. I. $-[v v]$ as a function of $\mathrm{N}$.

solution $A$ is the equivalent of my own solution $I$, the $N$-value of which (as determined by $\frac{\mathrm{I}}{m_{\oplus+c}}=328452$ ) is represented by the $\mathrm{I}$ in figure $\mathrm{I}$. It may be noted that between $I$ and $A$, or on the left of $A$, the slope of the curve is less steep than on the right of $\mathrm{A}$. 
While the shallowness of the minimum of the $[v v]$ curve at $\mathrm{A}$ explains the sensitivity of $m_{\Phi+c}$ and thus of the solar parallax against any interference with a ,, free " solution from Eros alone, the slope at C is already so steep, and the related value of $[v v]$ so far above the minimum at $\mathrm{A}$, that it seems far from justified to consider the solution $\mathrm{C}$ as one doing justice to the Eros observations. It should be noted that each residual of Eros is based on a normal place representing numerous individual observations. The only argument in favor of solution $\mathrm{C}$ is its agreement with the Venus radar determinations of the astronomical unit. The further argument, that solution $\mathrm{C}$ gives a sufficiently close representation of the Eros motion, has no real weight, because an infinite number of such solutions with even betler residuals exists on both sides of solution $\mathrm{A}$, as illustrated in figure $\mathrm{I}$. 